\title{
Autonomía de la mediación técnica y sistema técnico*
}

\author{
Franck Tinland $^{* *}$ \\ Traducción del francés al español \\ de Luis Alfonso Palau Castaño \\ DOl: 10.22395/csye.v6n11a10
}

El discurso sobre la técnica sigue siendo un discurso estallado en el que se yuxtaponen análisis etnológicos o históricos más o menos segmentados, apreciaciones éticas generalmente inspiradas en reivindicaciones humanistas, consideraciones sobre las interfaces entre técnica y sociedad. La Técnica misma rara vez es pensada a partir de sí misma, a partir de lo que la hace posible, según su modo propio de constitución y de desarrollo. Sin embargo, esto sería necesario para la comprensión de sus efectos de los que se sabe claramente que desbordan con mucho los productos que resultan de la operación de sus medios y procedimientos técnicos, y para la evaluación de la relación de ese desarrollo de nuestra potencia con el dominio que podemos tener sobre lo que condiciona nuestra existencia.

Las razones de esta situación son numerosas. En el horizonte de lo que hace de las técnicas los parientes pobres de la historia de nuestro pensamiento, por oposición especialmente a la teoría política o a la epistemología, es preciso ciertamente recalcar el descrédito que se ha echado sobre la manipulación vergonzosa, si no servil, de la materia. Pero conviene también tener en cuenta la falsa transparencia de las realizaciones de la actividad técnica. Esta se manifiesta ante todo en la proliferación de objetos visibles, tangibles, cuya existencia remite (no menos visiblemente), por una parte, a su utilidad, a la posibilidad de utilizarlos, y por la otra a la inteligencia de aquel o de aquellos que los han concebido antes de fabricarlos.

Pero la técnica es claramente otra cosa que lo que la panoplia de nuestras herramientas y máquinas pone de manifiesto, en la exhibición y la exterioridad mutua de los objetos de los cuales nos servimos según las situaciones que vivimos. La primera tarea, frente a la amplitud de un fenómeno que revela nuestra presente condición, pero cuya proveniencia es claramente más antigua, es la de señalar su coherencia propia y determinar sus correlaciones internas; en resumen, de interrogarla a ella misma, en su orden y en su desarrollo de

\footnotetext{
Tomado de Prades, Jacques (1992). La tecnociencia. Las fracturas de los discursos. París: L'Harmattan. Capítulo V: La autonomía de la técnica (pp. 121-149). Traducción del francés al español por Luis Alfonso Palau Castaño, Medellín, abril 2017. Nota del editor.

** Profesor de Filosofía en la Universidad de Montpellier. Autor entre otras de: La diferencia antropológica, 1977, y de Derecho natural, ley civil y soberanía en la época clásica, 1988. Co-autor de La tecnociencia en cuestión. Seyssel: Champ Vallon, 1990. Nota del traductor.
} 
realidad completa, en lugar de considerarla en su relación con los que inventan sus formas nuevas o la utilizan para sus fines propios.

Si hay una especificidad del orden técnico ella está en los vínculos constitutivos de dicho orden, en el tejido de las relaciones internas a todo lo que compromete la tecnicidad (herramientas, procedimientos, materiales, modos de adquisición de la competencia en la producción y el uso de los objetos técnicos) donde es necesario buscar esta unidad de organización y de sentido que constituye la técnica como objeto potencial de análisis y de reflexión, irreductible a cualquier otro.

Ahora bien, tal es claramente lo esencial: la importancia para nosotros del hecho técnico, la obligación de pensarlo en la plenitud de sus determinaciones y de sus efectos a pesar del poco interés que le demuestra nuestra tradición imponen lo que se presenta ante todo como exigencia metodológica e hipótesis cuya pertinencia hay que poner a prueba.

Esta exigencia y esta hipótesis conducen a llevar tan lejos como sea posible la explicación de lo técnico por el técnico, luego a precisar los límites de la inteligibilidad así producida y a determinar entonces -pero entonces solamente-las interfaces e interacciones entre lo que tiene que ver con la tecnicidad, por una parte, y lo que se relaciona con otras formas de actividades y de producción en el seno de las sociedades humanas.

Tenemos que sustraerlo del reproche de arbitrariedad antes incluso de precisar lo que se admite implícitamente en la hipótesis según la cual lo técnico debe ante todo explicarse por lo técnico y evaluar así sus consecuencias. Pues, sumado todo, ¿sobre qué apoyarse para conceder por adelantado algún crédito a la idea misma según la cual hay un orden técnico, una coherencia interna en lo que tiene que ver con la tecnicidad, y que esta especificidad abre la posibilidad de la constitución de la técnica como "dominio" o como "región" de objetividad, correlacionada con otros, sin duda, pero, ante todo, diferenciada, determinable como diferente? Que nos sea suficiente aquí con evocar lo que se lee en filigrana en la superposición de lecturas diacrónicas y sincrónicas de las producciones técnicas, del utillaje en el sentido más amplio.

A larguísimo término, la especificidad de la historia de las técnicas se traduce en un desarrollo gradual de la eficacia y de la diversidad en el utillaje del que disponen los hombres globalmente. Esta progresión, este aumento en potencia de los medios que le asegura a la actividad técnica su dominación sobre el mundo, no excluye ni los períodos de estancamiento, ni las diferencias de ritmo (yendo de unidades de tiempo pertinente del orden de la centena de millares de años a la década), ni la diversificación geográfica y las especializaciones locales, ni el 
desplazamiento de los focos de innovación. Pero ella permite, en función de la elección de los parámetros considerados, el establecimiento de esas curvas del mismo aspecto general y del mismo sentido que las que trazó A. Leroi-Gourhan para el paleolítico a partir del utillaje de sílex, que F. Meyer prolongó tomando en consideración los niveles de energía controlada, o la velocidad de los medios de transporte, y que se podrían, sin duda, reencontrar si nos interesáramos en las actuaciones de los medios de cálculo, del ábaco a nuestra última generación de ordenadores, pasando por la máquina de Pascal.

El estilo de este desarrollo fue presentado así por M. Daumas: "el desarrollo de los medios técnicos es el resultado de una experiencia colectiva acumulada sin cesar. Cada generación continúa heredando experiencias de todas las que la han precedido. En el dominio técnico el progreso es una suma. Contrariamente a lo que se constata en la historia de las ciencias, por ejemplo, las vías no han sido dudosas; en todo caso, no lo han sido mucho menos. La técnica parece en efecto haber seguido una curva ascendente continua sin tener que proponer soluciones inciertas. No parece incluso que haya conocidos períodos de estancamiento o de regresión. Esto se entiende naturalmente, si se encara el conjunto de la humanidad y no de los grupos de población limitados"1.

La singularidad de esta duración, comparada con la de todas las otras formas de actividades humanas, fue subrayada así por A. Leroi-Gourhan: "la adquisición técnica no se pierde, su transmisión está asegurada por fuera de las aventuras políticas (...) Esta sobrevivencia, de la que la humanidad saca su estado presente, opone la actividad técnica a todos los otros dominios de la actividad humana (...). La continuidad del esfuerzo técnico en el hombre hace de la tecnología una disciplina en la que los valores comunes al resto de la etnología no son sino parcialmente aplicables. Si se busca el parentesco real de la tecnología, hay que hacerlo por el lado de la paleontología, hay que orientarse hacia la biología en el sentido amplio"2.

El conjunto de los medios técnicos prosigue una evolución que puede ser muy contrastada según las épocas y los lugares, pero que persevera en un mismo sentido: el de una multiplicación, de una intensificación, de una economía de materiales, de una miniaturización con iguales prestaciones, de un aumento en la precisión, con conexiones eficaces de las que disponen los hombres en la apropiación -el acto de hacer propio algo- y en la explotación de su entorno (natural y humano, pues para nada hay diferencias entre las herramientas y las armas, de caza o de guerra).

\footnotetext{
1 Daumas, Maurice, Las grandes etapas del progreso técnico, México, Fondo de Cultura Económica, 1996.

2 André Leroi-Gourhan. Medio y técnica. Madrid: Taurus, 1988.
} 
Si ahora consideramos el utillaje técnico que opera en una sociedad determinada en un momento de su historia, la dependencia mutua de las herramientas es patente. El más común de nuestros instrumentos de cocina supone el funcionamiento de herramientas que sirvieron para fabricar herramientas y que presuponen otros instrumentos. De lo uno a lo otro todo concurre en los gestos que hacen operatorio el utillaje que caracteriza a una sociedad, en el seno de un medio técnico que condiciona la producción y el uso de cada uno de sus constituyentes.

Sería posible evocar las complejas sinergias de medios presupuestos por la antigua forja (que asocia al trabajo del metal, la curtiduría, la cerámica, la preparación del combustible, la explotación de los yacimientos minerales...), por el simple uso del automóvil (con su "medio asociado": carreteras, materiales de revestimiento, estaciones de servicios, redes de aprovisionamiento de carburante, redes de mantenimiento, señalización... sin evocar todavía las fábricas, sus dependencias con respecto a la producción de energía, etc.) o por la solidaridad de las "tecnologías avanzadas" necesarias para la construcción y el funcionamiento de una central nuclear.

Es una solidaridad de este tipo la que se reencuentra tras la anotación de G. Simondon según la cual sería posible "leer" en una simple aguja de acero salida de una fábrica inglesa de fines del siglo XIX características que permiten situarla y datarle con referencia al estado de la metalurgia del otro lado de la Mancha en aquella época. Cada producto es como un micro-cosmos en el que se proyecta y se expresa el mundo técnico necesario en su fabricación.

Según las dos dimensiones evocadas -la de un devenir que testimonia la "continuidad del esfuerzo técnico" que se manifiesta a través de las modalidades de desarrollo originales, y la del concurso de todo el entorno tecnológico en la fabricación de una producción particular- el orden técnico se diferencia de su contexto a partir de las relaciones que herramientas, procedimientos operatorios, habilidades y modalidades de transmisión de las competencias mantienen en el seno de lo que toma figura de "medio", y más precisamente, de "sistema técnico".

Aislada del contexto que le da su sentido de realidad perteneciente al orden técnico, una herramienta puede volverse pieza de colección, tener un valor estético, etnológico o arqueológico, para no hablar de las nuevas destinaciones prácticas que puede recibir -tal como el fuelle de forja que se volvió bar- pero deja de ser lo que era, componente de un conjunto en función del cual podía ser utilizado y tomar lugar en el concierto de los medios eficaces por los que pasa la relación de una sociedad con su entorno, en tanto que esta relación se efectúa bajo el signo de una empresa que modifica lo "dado" para sacar de allí 
un "producto", extrayendo del mundo lo que es posible de apropiarse a través de las operaciones de transformación, una "hechura".

Tales son las características más bien triviales a partir de las cuales se encuentra justificado el proyecto de poner a prueba la hipótesis según la cual, tan lejos como sea posible hacerlo, lo técnico deba explicarse por lo técnico, como realización hecha posible por el conjunto de los medios que el sistema condiciona, más allá de la reproducción y del uso de sus unidades constitutivas, la renovación a través de la actualización de potencialidades siempre reabiertas a partir de lo ya realizado.

Innovaciones endógenas e importadas, por recepción de tecnologías exteriores, tienen acá su papel, al mismo título que las relaciones de fuerza y la búsqueda de la competitividad económica, o las reacciones imaginarias y los fantasmas que se difunden en el contexto socio-cultural. Pero esto no le quita nada a la pertinencia del esfuerzo por constituir el orden técnico como dominio específico de estudios, en la medida en que este dominio es colocado bajo el signo de su tendencia a una sistematización que solidariza sus elementos constitutivos por medio del juego de las dependencias mutuas.

Es a enfoques complementarios más que antinómicos, de los que se organizan en sistema de los medios eficaces que conectan con el mundo, a lo que remiten términos como los de medio técnico y sistema técnico, empleados entre otros por A. Leroi-Gourhan y B. Gille. Es a una difusión de este modo interno de organización en el seno de un contexto, donde termina por jugar un rol de foco estructurador de las relaciones interhumanas, a lo que J. Ellul le ha dado el nombre de sistema técnico.

La consecuencia de esta dependencia mutua de las formas, procederes, modos operatorios y condiciones de adquisición de las competencias técnicas es la autonomía de lo que impone su coherencia propia, asociada a la interdependencia que da su unidad al contexto socio-histórico en el que se desarrollan todos los registros de actividades humanas.

Autonomía significa sumisión a su propia legalidad, a la legalidad sobre la base de la cual se edifica el orden por el que se manifiesta y dura una diferenciación con respecto a lo que, en torno suyo, permanece exterior. Ella implica pues una capacidad de escapar a una determinación directa por las condiciones externas y de responder conforme a su ser propio, a las variaciones, presiones y solicitaciones de las circunstancias (de lo que lo "circunscribe"). Esta respuesta es la de un sistema cuyas regulaciones internas estabilizan, y a través de la cual él tiende a preservar su diferencia, a mantenerse, es decir, a mantener su coherencia. 
Por ejemplo, un viviente homeotermo tiende a estabilizar su temperatura interna, a pesar de las variaciones térmicas de su medio, y esto no aislándose de este último sino, por el contrario, desarrollando y regulando sus intercambios con su medio. Un tal viviente puede ser llamado autónomo sin, por tanto, ser independiente de sus condiciones externas de existencia. Muy por el contrario, tiene necesidad de flujos perpetuos de intercambios con el mundo alrededor para mantener su propia identidad.

El conjunto formado por los medios técnicos no es ciertamente un ser viviente. Pero este, como aquel se comportan -en grados diferentes...-como sistemas en los que la interdependencia de las partes constitutivas constituye una red de determinaciones y de regulaciones que delimita el marco de las posibilidades de variación de cada elemento y de las potencialidades evolutivas que le están abiertas. Es en función de esta pertenencia a un conjunto caracterizado al mismo tiempo por la emergencia de capacidades relacionales nuevas con el mundo, como las modificaciones "locales" que afectan los elementos, como se los debe comprender.

La autonomía del orden técnico remite pues claramente a una coherencia interna fundamentada en la interdependencia de sus constituyentes, y esta coherencia refleja a la vez la solidaridad de las herramientas, prácticas, habilidades, y la capacidad de integrar a su dinámica propia las oportunidades que presenta el entorno, o de remediar las penurias u obstáculos ligados a las circunstancias.

Conviene situar este orden técnico, y medir las implicaciones de su autonomía con respecto a lo que prolonga, como con respecto a aquello de lo que parece una simple consecuencia sin realidad propia.

Lo que prolonga la técnica, y que ante todo ella asume a su manera, es la apropiación por parte de los organismos vivos de los recursos presentes en su medio, es decir, el esfuerzo para extraer del entorno lo que ellos podrán asimilar, lo necesario para su subsistencia, haciendo de ello su propia sustancia a través de diversas transformaciones. Todo ser vivo extrae de su medio material, energía e información gracias a la diferenciación y a la especialización de su superficie de contacto con el mundo. La evolución de las especies ha conducido a la diferenciación y especialización de los captores de información que son los órganos sensoriales y de los dispositivos de prensión en función de los cuales se estructuran las relaciones del viviente y el mundo, relaciones características de la especie al mismo título que la organización interna del cuerpo.

La herramienta parece casi contemporánea de la emergencia de la forma humana, es decir, de un tipo morfológico que reposa sobre la diferenciación de 
las funciones de locomoción y de prensión entre miembros anteriores y posteriores ya realizada en los australopitecos. En el momento de su aparición, la herramienta lo hace como "prolongación anatómica del cuerpo", y se inscribe en línea recta de la exploración de las posibilidades de "contacto eficaz" que realiza la evolución a través de la multiplicación y la especialización de los medios orgánicos de prensión.

La herramienta exterioriza los dispositivos de conexión con las cosas de los que dispone el cuerpo bajo forma de manos, de ganchos, de pinzas, de picos y de garras. No vamos a repetir acá a A. Leroi-Gourhan que evidenció este enraizamiento biológico de la tecnicidad. Esta, desde sus orígenes, aparece como un medio de intensificación de flujos de intercambios entre el viviente y su medio, y desde el comienzo, esta intensificación toma -con la morfología general del cuerpo humano (hace cerca de tres millones de años) - un estilo original a través de las etapas que tampoco vamos a indicar aquí.

Pero, si conviene subrayar este enraizamiento que se olvida demasiado fácil dado el vuelo de nuestras actuaciones tecnológicas, es, a la vez, para hacerle contrapeso a una facilísima referencia al "genio humano", y a la filiación de la herramienta a partir de la idea, y para subrayar cómo, a partir de dicho enraizamiento, lo que es del orden técnico se emancipa al estructurarse como sistema autónomo.

Decir que los medios técnicos se integran en un tal sistema es decir que ellos tienden a constituir una mediación coherente y evolutiva que toma lugar entre términos cuyas relaciones ella contribuye a determinar. Por definición, un medio es lo que ocupa un sitio intermediario, lo que está "entre" esos términos "extremos" y que le da forma concretamente a su relación. Los medios técnicos, y la mediación que ellos tejen no son solamente lo que vincula un estado dado de las cosas con la realización de lo que se anticipa como fin, ni lo que permite la satisfacción de la necesidad, o del deseo a través de esta realización. Mucho más allá, ellos son lo que estructura las relaciones de los hombres que viven en una sociedad dada, con su entorno, y de manera más general, con el mundo... en tanto que estas relaciones están colocadas bajo el signo de la búsqueda de un dominio eficaz que permita transformar (hacer pasar de una forma "impropia", en bruto, a una forma "apropiada") lo que está ya ahí como horizonte de posibilidades y halo de amenazas. A través de esta "apropiación", no son solamente las exigencias vitales ligadas a la subsistencia biológica las que reciben satisfacción, sino también las necesidades de intercambio, fundamento de la socialidad de tipo humano, en la medida en que es permitido pensar que el intercambio regulado hace aparecer la necesidad de bienes por transmitir, mucho más allá de las necesidades biológicas de consumo. 
Como lo hemos dicho ya, los medios técnicos mantienen entre ellos esas relaciones de condicionamiento mutuo que los ordenan en una mediación reticulada de la que una sociedad humana saca sus capacidades de explotar su entorno para extraer de él recursos y producir lo que es necesario para la subsistencia de los hombres, como para su propia existencia en tanto que tal.

Pero la explotación de estas posibilidades presupone la sumisión a las limitaciones estructurales que impone tanto el uso de las herramientas como el de las palabras, y las mediaciones engendradas por la actividad humana que retroactúa sobre los hombres mismos. Ellas informan al ser humano tanto como no le quitan sus determinaciones y su existencia. Todo lo que precede conduce a buscar en la herramienta misma, o más bien en el utillaje considerado como conjunto de formas objetivas y como red de condicionamientos recíprocos entre esas formas, las condiciones de inteligibilidad de los objetos técnicos, de sus características, de su producción, de su uso, y de sus perfeccionamientos o multiplicación.

Precisamos ante todo considerar que lo que aquí es esencial no es la novedad o la innovación, sino la conservación, la repetición y el lento trabajo que opera sobre lo adquirido según perspectivas cuya puesta en evidencia supone el retorno al pasado más o menos lejano. En efecto, más allá de la relación de la herramienta con el medio técnico del que es solidaria, está aquello que soporta la relación, es decir, la tradición, la transmisión de un patrimonio tecnológico (desde las industrias líticas). La técnica, por todas partes y siempre donde ella aparece como marca de la humanidad, al mismo título que el lenguaje, es más memoria que invención. Ella presupone la capacidad de apoyarse en sedimentaciones muy profundas para proseguir el desarrollo y la diversificación de las conexiones con el mundo, haciendo surgir materiales, sobre el fondo de indiferenciación primaria de lo que no es utilizable, a medida que se presenten procedimientos de apropiación que, a su vez, se prestan y se ajustan a otras aplicaciones posibles.

Nos queda entonces por comprender la mediación técnica como un conjunto de formas materiales (matérielles), de procedimientos, de materiales (matériaux), de usos solidarios los unos de los otros. Este conjunto es evolutivo. Ofrece constantemente nuevas posibilidades de conexión, de interferencia, de recombinación, un poco como (para decirlo rápidamente) el puente echado entre el movimiento lineal alterno y el movimiento circular por el modesto embielado de rueda constituido hacia el siglo XIII, que significó la superación de uno de los obstáculos más importantes descubiertos por B. Gille para el desarrollo de la mecánica griega, y que contiene en germen, según A. Leroi-Gourhan, el uso que se hará de él, muchos siglos después en nuestras locomotoras. Es siempre 
en el marco de un medio técnico ya presente, y a partir de posibilidades combinatorias en las que es rico, como son posibles el trabajo de la inteligencia, la aplicación de los conocimientos y la incitación económica.

Afirmar esto no es ni subestimar el rol de esta inteligencia, ni negar las determinaciones económicas del progreso técnico, ni excluir la importancia de las actitudes simbólicas o de las expectativas sociales. Es solamente constatar que el genio o la voluntad de los hombres no pueden realizar sino lo que es posible. Ahora bien, lo propio del sistema técnico es generar posibilidades. Todas no van a ser igualmente explotadas. Algunas lo serán más tarde, en contextos diferentes. Otras nunca lo serán, y ellas sin duda no son todas composibles. La realización de algunas se sitúan en la prolongación de los grandes movimientos evolutivos que testimonian algunas series de objetos técnicos. Otras están más aleatoriamente ligadas a las circunstancias. Pero de todas formas, es en el tejido de las relaciones internas a la esfera técnica donde se engendran y renuevan estas posibilidades.

Ellas aureolan, rodean las realizaciones, ellas mismas, salidas de realizaciones anteriores. Ciertamente que no hay lazo mecánico entre el pasado, el presente y el futuro. Pero todo perfeccionamiento de una forma técnica, toda transferencia de la utilización de una herramienta o de un procedimiento hacia nuevas aplicaciones, toda recombinación de medios técnicos hacen renacer un más allá de lo realizado, por el que el sistema técnico se abre hacia su propio desarrollo, hacia su diversificación y la intensificación de la influencia que le da al gesto eficaz que transforma el material.

Requeriríamos aquí reconstituir tanto la historia de las utilizaciones del movimiento circular en sus dos grandes variantes (masa y potencia por una parte, liviandad y velocidad por la otra) o aquella, más reciente, pero que presenta como en resumen la misma lección, de las abundantes aplicaciones de los rayos "láser" desde hace veinte años, para reencontrar una misma dinámica interna a la mediación por la que las sociedades humanas se reportan al mundo en tanto que ellas explotan sus recursos.

Sería posible entrar más adelante en la exploración de esta autonomía interna al sistema técnico, es decir, tanto a las regulaciones que conducen, por una parte, al ajuste de las formas y procedimientos en la unidad de un conjunto cuyos elementos llevan todas la marca, y por otra parte, a la dinámica del desarrollo, incluido aquí el que se da bajo sus formas cíclicas. Habría lugar así, de recordar primero las curiosas Reflexiones sobre la ciencia de las máquinas que Jacques Lafitte publicó en 1932, y en las que aparecen nociones como las de "linaje" y de "forma general de la serie", en relación con el "perfeccionamiento simultáneo de la organización general y de la estructura", antes de subrayar 
las concordancias que se pueden establecer (hecha abstracción de las escalas de tiempo) entre la exposición de la evolución del biface paleolítico, tal como la presenta A. Leroi-Gourhan, y el recuento que hace G. Simondon de las etapas seguidas por el motor de combustión interna. En los dos casos, aparece una verdadera "ortogénesis" que se traduce en una "adaptación a sí mismo" del objeto técnico, tendente a individualizarla a través de una evolución de aspecto auto-normada, estando la diferencia en que, en el primer caso, muchos centenares de miles de años separan los sílex achelienses toscamente tallados, de las almendras tan finas que nos han dejado los musterienses, y en el segundo caso, algunas décadas son suficientes para evidenciar tal evolución.

La unidad de la técnica humana no se la podría ilustrar mejor que por la puesta en consonancia de estos ejemplos. Aunque sería más simple evocar la "tendencia" constante de las series de objetos técnicos a la miniaturización, tal como se la encuentra desde la historia de los bifaces y de la industria lítica en general, a las de nuestros motores (con potencia igual) y de nuestras bombas. Los sucesivos perfeccionamientos le están como sugeridos, si no dictados, al técnico (technicien), por el propio tipo técnico (technique), y lo mismo ocurre con la transposición de las utilizaciones de un dominio al otro. Asimismo, podríamos, a escala del conjunto, evocar los grandes ciclos en función de los cuales se reestructura el medio técnico, siguiendo una lógica interna subrayada por Gilbert Simondon, especialmente a través de la descripción del paso de /1/ las industrias cautivas de la fuerza motriz provista por los flujos naturales <corrientes de aguas, regímenes de vientos...>, a /2/ "la era termodinámica" <s. $\mathrm{XIX}>$, y posteriormente /3/ a la preponderancia de la producción y del uso de la electricidad, vueltas posibles por el desarrollo de la metalurgia del estadio precedente /2/. Cada ciclo se desarrolla hasta crear las posibilidades a partir de las cuales el conjunto se reorganiza, convirtiendo en obsoletas las condiciones de su propia emergencia.

Todas estas consideraciones, que solo pueden ser evocadas sumariamente, validan la hipótesis según la cual, antes de pensar la técnica en su relación con otra cosa distinta de ella misma (como aumento de la potencia de medios eficaces para actuar sobre el mundo), conviene volverla a aprehender en su unidad, es decir, como sistema de medios subordinados a una misma norma interna (la eficacia, distinta de la utilidad), que obedecen a leyes internas de estructuración y que envuelven potencialidades específicas de desarrollo. De todo esto resultan, que se las sienta o no, limitaciones a las que la prosecución de sus objetivos obliga a los hombres a someterse.

$\mathrm{E}$, incluso, tenemos que ir más lejos. Ciertamente las herramientas son en sí mismas objetos inertes. Nuestras más sofisticadas máquinas estarían condena- 
das al embotamiento o a otras formas de corrosión sin la solicitud humana. Con mayor razón, los "individuos" que pueblan el sistema no se engendran los unos a los otros sin la operación de los que tienen por función darles la existencia. Pero conviene evitar oponer al sistema técnico lo que sería su "otro", o en todo caso el que permanecería frente a él en situación de perfecta exterioridad: los hombres. Estos no son lo que son, solo existen tal como son en relación de los unos con los otros, en relación con lo que los rodea, en relación con el mundo como horizonte sobre el que se destacan cosas y seres. Toda existencia es, esencialmente, co-existencia que se manifiesta a través de la multiplicidad de los intercambios, de las interacciones, de las modificaciones aportadas a los flujos por los que circulan materia, energía, e información. Los hombres están, cada uno y colectivamente, en el foco de múltiples interacciones e intercambios. Su existencia, en gran medida, toma forma en las relaciones multidimensionales con aquello y con aquellos que coexiste. El medio técnico es, así como la lengua que habla, aquello en lo que esta existencia concebida como co-existencia recibe sus determinaciones concretas.

De este modo, los hombres no están "enfrente" del sistema técnico, en una exterioridad esencial que sobreañade a lo que son características accesorias, accidentales. Sin duda que el medio técnico no existiría sin ellos. Pero ellos manifiestan su existencia en tanto que son partes que toman o reciben de ese sistema (y que son tomados por él). Las regulaciones de este los atraviesan u orientan sus actividades, sus pensamientos, sus relaciones con el mundo y con ellos mismos.

No solamente las formas, procedimientos y materiales, nutren sus representaciones y la conciencia que tienen de sí mismos, sino que también esos medios estructuran sus deseos y movilizan sus esfuerzos en torno a fines solidarios de la capacidad de realización que dan. Verdaderamente siempre solo queremos -vieja lección estoica retomada por Descartes quien, sin embargo...- lo que pensamos posible. Ahora bien, el progreso técnico, el aumento en la potencia de los medios renuevan el horizonte de las posibilidades más aún que estar en el principio de realizaciones nuevas. Esto se aplica tanto a los individuos como a las naciones; nadie (o casi...) ha hecho nunca un gran esfuerzo para ir a la Luna antes de que la potencia de nuestros cohetes permitiera pensar que tal salto era realizable. El deseo de vacacionar bajo el sol tropical es ciertamente bien cultivado por las agencias de viajes, pero esta promoción ella misma presupone la muy compleja red de medios técnicos entrecruzados, que ponen el sueño a seis horas de distancia de una casa que no sería ella misma lo que es sin el aparataje eléctrico del que disponen nuestras sociedades.

No somos menos humanos (abstracción hecha de toda evaluación moral) en tanto que partes comprometidas en este juego de medios, que cuando vamos al 
teatro, leemos a Platón o celebramos la Navidad. Esta estructuración de nuestras relaciones con el mundo, con nosotros mismos pertenece a lo que somos en el horizonte temporal de nuestra existencia, y que hace posibles las mediaciones cuyo origen coincide con la aparición del género homo. Tratándose de la mediación que les da a los gestos humanos una eficacia sin equivalente, su esencia sigue siendo la misma, no obstante el hecho de que sus manifestaciones sean bien diferentes según las épocas.

Son precisamente estas diferencias las que están en el principio de nuestras preocupaciones con respecto a la técnica, y que conducen a precisar su estatus, su modo de existencia y de desarrollo. Es también lo que nos lleva a descubrir su autonomía, a medir sus incidencias, a precisar sus límites y, eventualmente, a buscar controlar sus modalidades.

Autonomía no significa ni clausura, ni independencia, sino inscripción de un orden fundado en interacciones y regulaciones entre sus constituyentes en un juego de condiciones y de interdependencias.

Uno de los lugares -o de los momentos- privilegiados para asomarse a los fenómenos de interfaces entre técnica y contexto histórico en el que se realiza una nueva configuración de sus relaciones sería aquel en el que, sobre el fondo de continuidad de su desarrollo, la curva del desarrollo técnico presentó un punto de inflexión que ha podido ser interpretado como ruptura. Se trata de lo que aconteció con el advenimiento de los tiempos modernos, y del que seguimos siendo tributarios. Esa época, que vio nacer el sistema cognitivo que llamamos ciencia, vio hundirse la red de relaciones simbólicas anudadas entre el orden cosmológico, la autoridad política, las referencias teológicas, en el seno de una lectura del mundo geocentrada, con finalidad, jerarquizada, que les asignaba a las criaturas su lugar y les indicaba a cada una su condición y sus deberes.

Frente a una teología que afirma más la trascendencia del Creador y que preferirá ver la marca de la omnipotencia divina en la regularidad de las leyes del mundo más bien que en la repetición de las intervenciones providenciales, frente a una cosmología que no solamente destituye a la tierra de su posición central en el mundo sino que, además, descubre los estigmas del devenir en la cara del sol y abre los cielos al infinito de los espacios que aterrorizaban a Pascal, frente al cuestionamiento de los fundamentos tradicionales del orden político que condujeron al modelo artificialista de la autoridad presentado en el Leviatán de Hobbes, el edificio del conocimiento se reconstruye sobre nuevas bases. Una vez despachadas las causas finales, el sistema de los conocimientos apuntalado en nuevas reglas de validación de sus enunciados, asocia estrechamente la operación sobre los signos (con la prevalencia del álgebra y la llegada de las nuevas formas del cálculo), la manipulación de las cosas (con el dominio 
metódico de las condiciones de un proceso físico requerido por la experimentación), apoyándose para ello en la mediación de la medida (que hace homogéneos, por cuantificación, los resultados de la observación empírica y los datos numéricos aptos a las transformaciones abiertas por las fórmulas matemáticas). Simultáneamente, la intensificación de los intercambios y la simulación de la producción conducen al interés nuevo que este conocimiento les presta a las operaciones técnicas.

El vínculo entre ciencia y técnica es desde entonces consustancial a esta forma de conocimiento, aunque la intencionalidad misma subyacente a las prácticas pueda ser muy diferente.

Lo esencial de todo esto es que, en este contexto brutalmente reestructurado en un siglo y medio, el lento ascenso de la técnica en poderío en el seno de las sociedades que desvalorizaban la acción vergonzosa sobre la materia, se encontró liberada de un cierto número de obstáculos simbólicos y, por el contrario, en consonancia con un entorno social y cultural en el seno del cual las tendencias internas al desarrollo de los medios técnicos se encontraron en acuerdo con las solicitaciones y preocupaciones ligadas a las nuevas formas de conocimiento, de intercambios y de organización social.

El proceso acumulativo así desencadenado, en una especie de sinergia que, sin embargo, no fue suficiente para hacer desaparecer cualquier reserva con respecto al técnico, está en el principio a la vez de la potencia de las naciones de Europa occidental (luego de las que han aceptado su "racionalidad") y de la imbricación siempre más estrecha del conocimiento y de lo que está muy lejos de ser una simple "aplicación", la acción transformadora de las cosas, en el seno de la tecnociencia.

La borradura del concepto clásico de verdad frente al vínculo de los enunciados validados y de la operatividad subyacente a esta validación, la amplificación de las interacciones entre investigación "básica", imperativos industriales y exigencias militares han conducido a una situación en la que el saber sigue a la traza las posibilidades de manipulación de las cosas y ofrece a los procedimientos de transformación aplicados a los materiales, los medios de una renovación acelerada. Y esto hasta el punto de que se puede, sin duda, decir que el desarrollo técnico (del que se volverá a decir que engendra más posibilidades que realizaciones) y sus posibilidades, incluso fantasmáticas, tienen una eficiencia de primer plano, determina los imperativos y las elecciones del aparato productivo más que a la inversa; en un sentido la Técnica "avasalla" a la economía gracias a la forma y al ritmo de la proliferación de los "medios" que pone a disposición de los agentes económicos. ¿Cómo entonces, salvo intervención externa, descuidar lo que es más impuesto que ofrecido? 
La historia reciente de los medios al servicio de la grabación musical ofrecerá, ella sola, una ilustración de las limitantes que la tecnología hace pesar sobre la viabilidad de las empresas que rehúsan las conversiones impuestas por la emergencia de nuevas técnicas. El tener en cuenta lo que se engendra en el corazón del sistema técnico es acá un imperativo que la apariencia de las escogencias libres -es decir, elecciones "inteligentes" que coincidan con la dinámica tecnológica del momento- enmascara bajo la tranquilizadora satisfacción de saber captar la ocasión, y darse una ventaja sobre los competidores.

Todos sabemos, mientras tanto, que la rentabilidad no siempre hace buenas migas con la rapidez de la evolución tecnológica y el progreso de las prestaciones a las que ella les abre la vía.

Tenemos que estas consideraciones sobre el estilo del desarrollo tecnológico en los tiempos modernos invocan otras. Deben, ante todo, ser vueltas a colocar sobre el fondo de ese largo término que es el único que permite medir lo que se juega tras el despliegue de los medios técnicos y de la potencia engendrada por el sistema que ellos forman. Cualquiera sea la distancia que nos separa de las industrias paleolíticas, es en la misma condición fundamental y en la unidad de un mismo proceso en donde se constituye la red de medios que lleva consigo potencialidades organizacionales y dinámicas, más o menos pronto explotadas o reconocidas. Ellas están enraizadas en la búsqueda de eficacia gracias a dispositivos que forman un conjunto coherente, abierto a la diversificación, la precisión, la potencia al menos potencialmente perfectible de un utillaje del que ninguna sociedad está totalmente exenta.

Las consecuencias de ello son, ante todo, que los hombres, cualquiera sea su posición con respecto a la puesta en funcionamiento de esos medios, solo tienen una conciencia muy fragmentaria de aquello en lo que participan, de lo que contribuyen a hacer existir y a desarrollar. De la misma manera que los locutores no saben todo lo que requiere su palabra y a cuáles reglas ellos obedecen, o que los agentes económicos no son conscientes de los mecanismos y de las regulaciones de los que se sirven, o a los que ellos sirven -recuérdese no más la metáfora de la "mano invisible"-, artesanos, ingenieros, bricoleres o simples usuarios de instrumentos cotidianos no pueden medir las condiciones y consecuencias de sus gestos, en tanto que estos se inscriben en la continuidad esencial de un proceso autonomizado, a la vez, con relación al orden de la vida y con respecto a las voluntades y proyectos de los individuos y de las comunidades. Las perspectivas que los unos y los otros tienen sobre lo que hacen permanecen truncas, confusas, limitadas al cortísimo término.

La misma inadecuación se reencuentra en las representaciones y elaboraciones teóricas (o ideológicas) a través de las cuales se encuentran reflexionadas 
las prácticas técnicas y por las que son evaluadas (o devaluadas). De este modo, cuando, por ejemplo, hablamos de la técnica en la Grecia antigua -y a menos que sigamos el esfuerzo de B. Gille para interesarnos en esta tecnología "naciente"3_ discurriremos sobre el discurso de autores, sin duda, poco habituados a las herramientas y a los procedimientos constitutivos de esta técnica; podemos estar seguros de que su visión no excedería la de nuestros propios ingenieros...

Participamos en la prolongación de una aventura que comenzó con homo habilis, si no antes. De esta participación tenemos una visión tan miope como la de nuestros predecesores, o casi... Nuestra situación es, sin embargo, nueva. Experimentada de forma confusa, esta novedad nos acarrea actitudes contradictorias, a la vez herederas del optimismo del que fue Saint-Simon el más elocuente de los heraldos, y de angustias cuya antigüedad recuerda viejos mitos. Pero esta novedad nos impone tomar en serio la técnica en tanto que modo de organización específica de una realidad que no se reduce a la objetivación de nuestros proyectos o a la concretización de nuestros conocimientos.

Esta novedad podría y debería analizarse bajo múltiples aspectos. Ella concierne en lo esencial no tanto el devenir mismo de las técnicas sino a las potencialidades y a los efectos de su puesta en funcionamiento, es decir, lo que inducen en su contexto. Este contexto presenta dos caras, que no podrían ser disociadas, o más bien, cuya imposible disociación es ella misma una consecuencia del estilo de nuestro medio técnico. La primera de esas caras concierne las relaciones entre las sociedades humanas, y en cada una de ellas, entre los propios hombres. La segunda concierne a las relaciones con el mundo, o la naturaleza.

Las distorsiones ligadas a la relación de los hombres con las técnicas contemporáneas están en el principio de desequilibrios crecientes, ligados al diferencial de desarrollo tecnológico, a la vez efecto y causa de las modalidades de acceso a los medios y circuitos económicos. Cada quien puede medir, localmente o a escala de la tierra, los peligros ligados a tales desequilibrios.

La segunda cara concierne a la inminencia de las diversas amenazas -aquí igualmente locales y globales- de orden ecológico. Estas están ligadas a las modalidades de explotación del medioambiente por la mediación de un sistema técnico cuyas interacciones con los sistemas naturales han dejado de ser despreciables ante aquellas sobre las que reposan los grandes equilibrios constitutivos de la biosfera, y las regulaciones por las que ella superpone su orden propio a las condiciones cosmo-físicas que son las del planeta.

Es la razón por la que, antes incluso de poner de relieve la novedad de nuestras capacidades y la especificidad de nuestras tecnologías, conviene situar este

3 B. Gille. Los mecánicos griegos. El Nacimiento de la tecnología. París: Seuil, 1980. 
suceso en el horizonte de la larga duración; el que ve la emergencia simultánea de la manera de existir propia de los hombres y de esos sistemas mediadores a través de los cuales se reorganizan las relaciones de este viviente singularmente organizado con la naturaleza.

La consideración de ese largo término, y el reconocimiento de las diversas manifestaciones de la autonomía propia del sistema de los medios técnicos conducen a las consecuencias ya subrayadas. La primera de ellas es que los hombres, en tanto no dispongan de los medios para perseguir fines previamente asignados, están dispuestos en este juego de posibilidades y de realizaciones, a ser, según el caso, productores, utilizadores o servidores de ese conjunto. Son solidarios con él en tanto que se someten a sus constreñimientos inherentes a la eficacia y que desearán aquello cuya realización permite esperar los medios técnicos.

La segunda es que el despliegue del abanico de las conexiones con la realidad física, biológica y humana depende muy poquito de su decisión y no se manifiesta para nada a su conciencia, a no ser de forma trunca y como fuente de ilusiones.

La tercera es que al nivel de las capacidades de acción y de la potencia de transformación inherentes a la técnica contemporánea, lo que era percibido como promesa de un porvenir mejor -sin que tengamos que renegar de él- deja emerger sordas inquietudes frente a realísimos efectos de desestabilización de los sistemas sociales y naturales cuyos equilibrios relativos (o las capacidades de reequilibramiento) condicionan una existencia humana aceptable, si no queremos decir "normal".

Frente a estas consecuencias de una validación de la hipótesis según la cual hay una autonomía del orden técnico, es necesario recordar la distancia que separa autonomía e independencia o auto-suficiencia.

La autonomía del orden técnico no es incompatible con los controles sociales que se ejercen espontáneamente sobre todos los tipos de actividades de los que "vive" una sociedad. Toda sociedad integra el sistema de sus medios de acción eficaces sobre el entorno en una red de representaciones y de instituciones que, sin hacerle perder sus caracteres específicos, encuadra su desarrollo y bloquea la realización de algunas potencialidades. Los límites impuestos -pero quizá de manera precaria- a la tecnología bio-médica manifiestan en nosotros una resistencia del cuerpo social que, antaño, se opuso a la disección del cadáver humano.

No es menos cierto que desde hace tres siglos -poca cosa en comparación con los tiempos en que se constituyeron las condiciones de nuestra propia potencia sobre la materia- la técnica se ha impuesto progresivamente como el lugar de 
las apuestas importantes en función de las que se ordena la existencia de los hombres, las modalidades de su coexistencia sobre el horizonte de mundos.

Nuestra forma específica de existencia se ha colocado bajo el signo de la eficacia en la apropiación activa de las condiciones naturales y sociales de las que ella depende, y por tanto de operaciones de transformación y de reorganización que requieren y que engendran potencia y competencia. La relación cognitiva con el mundo se ha integrado de manera progresiva en el conjunto tecno-científico, de la misma manera que la competencia económica y los enfrentamientos en el campo de batalla son tributarios de las capacidades de acción, de intervención, de transformación desarrolladas bajo múltiples formas en el seno de la mediación técnica. Los esquemas fundamentales que subtienden la competencia técnica -la racionalidad técnica, si se quiere- se han difundido y difunden en el contexto social y cultural. Este proceso que asegura la empresa del sistema técnico sobre sus condiciones externas, sobre sus entornos, es lo que pone en escena J. Ellul bajo el nombre de "sistema técnico". Si esta descripción es buena, entonces, es verdad: autonomía significa no independencia sino subordinación, "avasallamiento" de todo aquello con lo que la mediación técnica mantiene inevitables interacciones.

La cuestión final es pues claramente saber hasta dónde la organización y el desarrollo autónomo del sistema de los medios técnicos pueden llegar en la extensión de sus regulaciones y referencias internas sobre el conjunto de sus condiciones, es decir, de la vida social y simbólica. La respuesta condiciona la posibilidad de una dominación humana del desarrollo técnico.

La exigencia y la legitimidad de esta dominación derivan de dos fuentes esenciales. La primera es el mantenimiento de la polivalencia, o de la multidimensionalidad de la existencia humana. Manifiestamente existen otras formas o facetas de la coexistencia de los hombres entre sí, con la naturaleza, con el mundo y su más allá, que las que pasan por la búsqueda de una empresa eficaz en la apropiación y organización de los entornos. No menos, indiscutiblemente, esto sería un empobrecimiento de las potencialidades de coexistencia como medir su valor con el patrón de la racionalidad técnica.

La segunda de estas fuentes tiene que ver con los límites de la expansión de este sistema -asimilable a una estructura altamente disipativa- en un contexto humano y natural cuya estabilidad y viabilidad reposan sobre equilibrios cuya fragilidad revela nuestra potencia.

No podemos pecar ni por exceso de irenismo ni por catastrofismo. Los recursos de la "creatividad humana" (que pueden manifestarse por lo demás bajo las formas más discutibles, como producciones simbólicas remitiendo a 
una credulidad infantil) son de una naturaleza tal como para hacer resurgir la multidimensionalidad de la existencia en los márgenes siempre renacientes de un sistema técnico en expansión... Sin duda que serían más preocupantes, y que ya lo son, las alteraciones del medio terrestre, así solo sea bajo la forma -anodina en apariencia puesto que diseminada y polimorfa- de los desechos, es decir: de lo que las actividades de transformación generan en la prosecución de la apropiación del mundo, al mismo título que los "productos" a los que solo se les presta atención.

Sin embargo, ha habido tanto grito de alarma lanzado antaño ante los efectos anticipados de técnicas hoy superadas, que conviene ser prudentes en los juicios que se lancen sobre los cambios esperados. No puede ni debe ser menospreciada la capacidad misma de adaptación del medio técnico en la prolongación de la búsqueda de un dominio sobre el entorno y sus propios mecanismos reguladores.

Además, las dos fuentes en las que se origina la obligación de un control del desarrollo técnico en función de otras referencias distintas a las normas internas al sistema de medios (la eficacia transformadora y reordenadora) corresponden a una condición bien real, que es la de nuestra actualidad. ¿Cómo concebir pues el reequilibrio de las tendencias hegemónicas ligadas a la autonomía del sistema técnico gracias al juego de regulaciones que sustituyen el control social espontáneo, debilitado por la centralidad del fenómeno técnico por todas partes donde son evocadas -con un término que implica respeto-las "tecnologías de punta"?

Este reequilibrio solo puede venir aquí (a la inversa del control que ejerce un contexto tradicional) de un proyecto formulado claramente frente a un porvenir anticipado, que da sentido a los anhelos, aceptaciones, rechazos. Y esto equivale a decir que la autonomía del sistema técnico, que tiende a la realización de sus potencialidades a través de la intensificación del "contacto eficaz" y la diversificación de su "superficie de contacto" con el mundo gracias a la reabsorción de los "cuellos de botella" que aparecen en su seno (defectos de materiales adecuados, procedimientos poco eficientes, niveles energéticos que se revelan insuficientes, carencia de competencia humana, deficiencia en la inversión...) debe ser encuadrada por dispositivos dispuestos para meter en cintura e incitar conscientemente.

Esta puesta en su lugar debe ser decidida. Y solo se lo puede hacer sobre la base de estudios prospectivos y de elección fundamentales, de una naturaleza tal que comprometa el devenir de nuestras sociedades. Esto exige, a la vez, experticias especializadas en las que se expresen las capacidades del sistema técnico mismo, y debate público. Se trata de la elaboración de una política, en el sentido más fuerte del término. 
Ir más adelante en este sentido nos sacaría del marco de las presentes reflexiones. Pero si hay autonomía del orden técnico, y si esa autonomía tiende a escapar a su integración en un conjunto multidimensional y "multi-normado" que delimitaría sus funciones con respecto a otros objetivos, y que tiende a una hegemonía ciega a toda referencia externa a la suya, entonces la anticipación de sus consecuencias y la orientación de su curso solo pueden venir de una toma de conciencia de los individuos y de la opinión, por tanto, de un debate nutrido de informaciones y capaces de desembocar en escogencias. La conciencia es, esencialmente, ese medio de integración y, por tanto, subordinación de lo parcial, que se desarrolla cuando la dominancia o la presión de una componente de la existencia amenaza el equilibrio, y que conduce a encargarse de lo que hasta entonces tenía más que ver con la espontaneidad y con el automatismo.

Tenemos pues que la técnica, en sus formas y ritmos presentes, produce más posibles de los que le es posible realizar a nuestras sociedades. Así sea por razones económicas, ninguna comunidad humana está en capacidad de actualizar las potencialidades con las que las tecnologías contemporáneas abren y renuevan el horizonte. La economía es aquí un factor limitativo de la expansión técnica, y esto implica elecciones (que son exclusiones) tanto como orientaciones, aunque haya que permanecer prudentes en lo que concierne a opciones y rechazos. El ejemplo de los supersónicos comerciales y de un futuro del que no están excluidos a perpetuidad, podría propiciar meditaciones, inciertas. Sin duda que es por intermediación de las decisiones presupuestales que tienen que ver con la potencia pública, como se podría percibir mejor las condiciones de lo que podría ser una verdadera política de regulación tecnológica, que actúe sobre las condiciones externas a las que debe ajustarse el sistema técnico, del que, sin embargo, su autonomía garantiza la imprevisibilidad de las respuestas más allá de un cortísimo término.

Es pues real que las formas presentes del sistema técnico tienden a revestir -más allá de la autonomía interna que le da a la técnica sus caracteres específicos en el seno de las actividades humanas- un carácter hegemónico, que transforma en sistema técnico el conjunto de nuestras condiciones inmediatas de existencia. Todo sistema en desarrollo, en el que predominan las retroacciones positivas, extiende su imperio y ejerce su poder regulador sobre estas condiciones, "internalizando" lo que permanecía en sus márgenes y que limitaba, a veces de forma aleatoria, su funcionamiento optimizado. La historia del sistema de nuestros medios técnicos o la historia vista desde el punto de vista de ese sistema ilustraría esta expansión por la que los medios de nuestra eficacia extienden su eficiencia sobre todo aquello de lo que dependen, incluso suscitando la neutralización de los efectos negativos de su empleo. 
Pero esto no excluye el esfuerzo conscientemente asumido para preservar lo que él excluye o amenaza, con referencia especialmente a las dos fuentes evocadas aquí antes. El rol de la toma de conciencia es precisamente abrir el horizonte de una integración y, por ende, de una subordinación, de lo que amenaza, por su dinámica propia, el equilibrio de las funciones y de los objetivos en su pluralidad y multidimensionalidad.

La autonomía de las técnicas no solamente no excluye sino que convoca este proceder, por múltiples razones, pero, ante todo, porque proviene de la necesidad para la continuidad misma de las actividades humanas, preservar las grandes constantes y los equilibrios mayores que son solidarios de nuestra vida y de nuestro bienestar.

Que sea así no significa que sea fácil responder a estos imperativos. Toda nuestra organización económica y política se ha ido centrando progresivamente, desde hace tres siglos, en las operaciones de transformación en vía de expansión acelerada, y sobre el valor que ellas añaden a lo que no tenía precio o interés más que en la medida en que eso podía ser considerado como material para trabajar, es decir, que entraba en la órbita de la actividad que se ejercía por la mediación de los medios técnicos. Son pues considerables las resistencias que hay que vencer para introducir otras "referencia" al "pilotaje" -etimológicamente, al gobierno- de las sociedades de alto potencial tecnológico. La obligación de vencerlas, sin embargo, nace de la potencia misma de nuestros medios.

Es verdad que esta es quizá la última astucia del orden técnico: hacer manifiesta la obligación de velar por su orientación, para asegurar mejor un desarrollo que cuestione la destrucción de sus propias condiciones de posibilidad.

No llegaremos hasta esta lectura extrema de la autonomía de la técnica. Pertenece a las características esenciales de nuestro ser el trascender, por la representación de lo actual y de lo posible, la red de determinaciones que estructuran nuestra manera de existir en el seno de un mundo con el que nuestras relaciones permanecen abiertas, sobre un fondo de imprevisible futuro. Es también esto lo que fundamenta nuestra responsabilidad... 